\title{
Role of the MDM2 Promoter Polymorphism (-309T>G) in Acute Myeloid Leukemia Development
}

\author{
Anuradha Cingeetham ${ }^{1}$, Sugunakar Vuree ${ }^{1}$, Sangeeta Jiwatani ${ }^{2}$, Sailaja Kagita ${ }^{2}$, \\ Nageswara Rao Dunna ${ }^{4}$, Phanni Bhushann Meka ${ }^{1}$, Manjula Gorre ${ }^{1}$, Sandhya \\ Annamaneni ${ }^{1}$, Raghunadharao Digumarti ${ }^{2}$, Sudha Sinha ${ }^{3}$, Vishnupriya Satti ${ }^{1 *}$
}

\begin{abstract}
Background: The human homologue of the mouse double minute 2 (MDM2) gene is a negative regulator of Tp53. MDM2-309T>G a functional promoter polymorphism was found to be associated with overexpression thereby attenuation of $\mathrm{Tp53}$ stress response and increased cancer susceptibility. We have planned to evaluate the possible role of MDM2-309T $>\mathrm{G}$ polymorphism with risk and response to chemotherapy in AML. Materials and Methods: A total of 223 de novo AML cases and 304 age and sex matched healthy controls were genotyped for the MDM2-309T>G polymorphism through the tetra-primer amplification refractory mutation system (ARMS)-PCR method. In order to assess the functional relationship of -309 T > G SNP with MDM2 expression level, we quantified MDM2 mRNA in 30 primary AML blood samples through quantitative RT-PCR. Both the (-309T>G) genotypes and the MDM2 expression were correlated with disease free survival (DFS) rates among patients who have achieved complete remission (CR) after first induction chemotherapy. Results: MDM2-309T>G polymorphism was significantly associated with AML development $(\mathbf{p}<\mathbf{0 . 0 0 0 1})$. The presence of either GG genotype or $\mathbf{G}$ allele at MDM2-309 confered $1.79(95 \%$ CI: 1.12-2.86; $p<0.001)$ and 1.46 fold $(95 \% \mathrm{CI}$ : $1.14-1.86 ; p=0.003)$ increased AML risk. Survival analysis revealed that $\mathrm{CR}+\mathrm{ve}$ cases with GG genotype had significantly increased DFS rates (16months, $\mathrm{p}=0.05)$ compared to CR+ve TT (11 months) and TG $(9$ months) genotype groups. Further, MDM2 expression was also found to be significantly elevated in GG genotype patients $(p=0.0039)$ and among $C R+v e$ cases $(p=0.0036)$. Conclusions: The MDM2-309T $>G$ polymorphism might be involved in AML development and also serve as a good prognostic indicator.
\end{abstract}

Keywords: AML - MDM2 - tetra primer (ARMS) PCR - RT-PCR - disease free survival - complete remission

Asian Pac J Cancer Prev, 16 (7), 2707-2712

\section{Introduction}

Acute myeloid leukemia (AML), a hematological malignancy, results due to failure of cell surveillance mechanism in eliminating mutant hematopoietic stem cells which undergo leukemic transformation with uncontrolled propagation. The variations in genes controlling cell surveillance mechanisms can influence or impair the constitutive capacity of the host cells in dealing with DNA lesions which potentially increases individual susceptibility to AML development. In general, the fate of mutated cells is decided by crucial protein Tp53, that selectively guides them either for repair and survival, or apoptosis (cell suicide programme) based on the extent of DNA damage. Many types of cancers had exhibited inactivation or attenuation of p53 gene expression either due to mutations or abnormal expression of p53 regulators, such as MDM2, the human homologue of the mouse double minute 2 (Michael and Oren., 2002), an oncogene that negatively regulates TP53 (Boyd et al., 200). Transcription of MDM2 is activated by TP53 which inturn leads to the degradation of TP53. A normal interaction between the two proteins allows TP53 to be maintained at low levels in normally dividing cells. Frequent inactivation of TP53 signaling through MDM2 over expression might lead to escape of malignant cells from apoptosis and progress into leukemia.

Human MDM2 gene is located on chromosome 12q14.3-q15 (Oliner et al., 1992) and comprises of 12 exons with two distinct transcriptional promoter elements P1 and P2 (Barak et al., 1994; Zauberman et al., 1995). The P1 promoter controls basal expression of MDM2, and is situated upstream in first exon of the MDM2 gene. The P2 promoter, located in the first intron is highly regulated and responsible for inducible expression of MDM2 (Montes de Oca Luna et al., 1996). The p53 response elements are located upstream of the $\mathrm{P} 2$ promoter, and hence the p53-inducible expression of MDM2 can be monitored

${ }^{1}$ Department of Genetics, Osmania University, ${ }^{2}$ Nizam's Institute of Medical Sciences, ${ }^{3}$ MNJ Institute of Oncology Regional Cancer Center, Hyderabad, ${ }^{4}$ School of Chemical and Biotechnology, SASTRA University, Thanjuvur, India *For correspondence: sattivishnupriya@gmail.com 
by expression levels of this specific transcript. There is also evidence that these two transcripts may differ in their propensity for alternative splicing as well as in their translatability (Brown et al., 1999; Cheng et al., 2007). A promoter polymorphism at $-309(\mathrm{~T}>\mathrm{G})$ position in MDM2 gene involving the substitution of T nucleotide by $\mathrm{G}$ results in increased affinity for transcription factor Sp1 and subsequent overexpression of MDM2 mRNA and MDM2 protein, thereby leading to attenuation of the p53 stress response (Bond et al., 2004). Since this polymorphism was studied exclusively as a potential risk factor in the development and progression of different cancers among various ethnic groups (Ellis et al., 2008; Phillips et al., 2010; Ebid et al 2012 ), our study was aimed to investigate the association of MDM2-309T $>$ G promoter polymorphism with the development and treatment outcome of AML patients from Southern India and also the functional relationship of this SNP with MDM2 expression level.

\section{Materials and Methods}

\section{Study subjects}

The present study includes 223 de novo AML cases recruited from Nizams Institute of Medical Sciences (NIMS), Hyderabad and Mehdi Nawaj Jung (MNJ) hospital and Cancer Regional Center, Hyderabad, India between 2006 and 2011. Clinical data including age at onset, Hemoglobin $(\mathrm{Hb}) \%$, white blood cell (WBC) count, platelet count, bone marrow blast $\%$ at diagnosis was collected. The information on complete remission rate (CR) and duration of disease free survival (DFS) time was collected after followup with the help of a Medical oncologist. For case-control comparison, 304 age and sex matched healthy controls from local population were randomly selected. All the controls who accepted to participate in the study had undergone a systematic interview to evaluate personal and family history of any type of cancers. Those who had a positive family history of cancers were excluded from the study. All AML patients and controls had provided written informed consent for testing on DNA samples. In case of minor subjects ( $<18$ yrs) informed consent was signed by the parents or guardians. The Institutional ethical committees of Nizams Institute of Medical Sciences (NIMS), Hyderabad and Mehdi Nawaj Jung (MNJ) hospital and Cancer Regional Center, Hyderabad and Osmania University, Hyderabad, India had approved the present study. Moreover, a personal interview was conducted using a structured questionnaire to collect information on epidemiological variables (age, gender, occupation, habits and habitats, etc.) for all the participants. In addition, each participant had provided $5 \mathrm{ml}$ of peripheral blood sample in ethylene diamine tetra acetic acid (EDTA) vaccutainer which was stored at $-20^{\circ} \mathrm{C}$ freezer until use.

\section{MDM2 Genotyping}

Genomic DNA was extracted from peripheral blood samples using a conventional DNA isolation protocol by Lahiri (Lahiri and Nurnberger 1991). The purity and quantity of the extracted DNA was checked by NanoDrop
(TM) 1000 UV/VIS Spectrophotometer (Thermo Fisher Scientific, Waltham, USA) and normalized to $50 \mathrm{ng} / \mu \mathrm{L}$ as a final concentration. The genotypes of the MDM2309T>G (rs2279744) polymorphism was determined by a tetra-primer amplification refractory mutation system (ARMS)-PCR method which uses two primer sets as described previously (Xiao et al., 2010). To cross validate, about $15 \%$ of the samples were randomly selected and regenotyped by a laboratory personnel who was unaware of the clinical status of the subjects and found to be in $100 \%$ concordance.

\section{MDM2 $m R N A$ real time assay}

In order to evaluate the effect of MDM2 -309T $>\mathrm{G}$ polymorphism on MDM2 expression in AML cases, we have quantified MDM2 mRNA level in 30 primary AML blood samples through Quantitative RT-PCR. We have used Taqman chemistry on an ABI Step One Real Time PCR machine (Applied Biosystems). The peripheral blood samples from 30AML patients were used for total RNA isolation using TRIzol reagent (life technologies, Carlsbad, USA, CA92008) and converted into cDNA using high capacity cDNA reverse transcription kit (Applied Biosystem, Foster City, CA94404). $\beta$-actin was used as an endogenous reference gene (Hs99999903_m1) for relative quantification of MDM2 mRNA expression (Hs00242813_m1) which was carried out in triplicates.

\section{Statistical analysis}

We have used SNPSTAT online tool to determine significant differences in genotype frequencies between cases and controls and to calculate Chisquare and Odds ratios. SPSS software, version 20.0 was used to calculate mean and standard deviations as well as Kaplan-Meier curves from data on survival analysis to evaluate association of genotypes with Disease free survival (DFS). Disease free survival (DFS) was defined as the time from the date of attaining the leukemia free- state or CR after primary induction treatment to the date of acute leukemia relapse or death from any cause, whichever occurs first. Survival analysis was performed only in complete remission (CR) cases, where relapse (or) death was censored as event. Differences in DFS rate between groups were compared through log rank test. Graphpad prims 5.0 software was used to construct dot graphs and ANOVA was performed to check the difference between the mRNA expression levels among different groups. A p-value of $\leq 0.05$ (two-sided test) was considered to be statistically significant.

\section{Results}

\section{Clinical characteristics}

The baseline clinical characteristics of 223 AML patients comprises of 118 males (52.9\%) and 105 females (47.1\%), whose mean age at diagnosis was found to be $30.90 \pm 14.28$ years (range, 4-75years). The mean values of different clinical characteristics like WBC count, hemoglobin level, platelet count, peripheral blast \% and LDH level were provided in Table 1. After first induction chemotherapy, data on remission rate could be collected 
Table 1. Genotype Distribution of MDM2-309T $>$ G Polymorphism and Baseline Clinical Characteristics at DiagnosisAML ( $\mathbf{N}=\mathbf{2 2 3})$

\begin{tabular}{|c|c|c|c|c|c|}
\hline \multirow[t]{2}{*}{ Variable } & \multicolumn{4}{|c|}{$\operatorname{AML}(\mathrm{N}=223)$} & \multirow[b]{2}{*}{$\mathrm{p}$} \\
\hline & $\mathrm{N}(\%)$ & TT & TG & GG & \\
\hline Gender & 223 & $50(22.42)$ & $76(34.08)$ & $97(43.50)$ & \\
\hline Males & $118(52.91)$ & $28(23.7)$ & $35(29.7)$ & $55(46.6)$ & 0.34 \\
\hline Females & $105(47.09)$ & $22(20.9)$ & $41(39.0)$ & $42(40.0)$ & \\
\hline Age at onset (yrs) & 223 & $50(22.42)$ & $76(34.08)$ & $97(43.50)$ & \\
\hline Mean \pm SD & $30.91 \pm 14.27$ & $29.82 \pm 14.75$ & $29.71 \pm 14.90$ & $32.40 \pm 13.50$ & 0.38 \\
\hline$>30 \mathrm{yrs}$ & $100(44.84)$ & 21(21.0) & $33(33.0)$ & $46(46.0)$ & \\
\hline$<20 \mathrm{yrs}$ & $47(21.08)$ & $11(23.4)$ & $20(42.6)$ & $16(34.0)$ & 0.6 \\
\hline 20-30yrs & $76(34.08)$ & $18(23.7)$ & $23(30.3)$ & $35(46.1)$ & \\
\hline $\mathrm{WBC}$ count $/ \mathrm{mm}^{3}$ & 223 & $50(22.42)$ & $76(34.08)$ & $97(43.50)$ & \\
\hline Mean \pm SD & $53369.19 \pm 77494.13$ & $56064.00 \pm 74126.6$ & $52750.00 \pm 79539.16$ & $52465.26 \pm 78327.93$ & 0.96 \\
\hline $4000-11000 / \mathrm{mm}^{3}$ & $64(28.70)$ & $17(26.6)$ & $22(34.4)$ & $25(39.1)$ & \\
\hline$<4000 /$ cumm & $15(6.73)$ & $1(6.7)$ & $4(26.7)$ & $10(66.7)$ & 0.46 \\
\hline $11000-30000 / \mathrm{mm}^{3}$ & $61(27.35)$ & $11(18.0)$ & $23(37.7)$ & $27(44.3)$ & \\
\hline$>30000 /$ cumm & $83(37.22)$ & $21(25.3)$ & $27(32.5)$ & $35(42.2)$ & \\
\hline Platelet count $/ \mathrm{mm}^{3}$ & 223 & $50(22.42)$ & $76(34.08)$ & $97(43.50)$ & \\
\hline Mean \pm SD & $00626.12 \pm 126479.68$ & $119780.00 \pm 146267.62$ & $104527.63 \pm 130890.82$ & $87696.12 \pm 110980.20$ & 0.33 \\
\hline$>1.5 \mathrm{Lakhs} / \mathrm{mm}^{3}$ & $46(20.63)$ & $14(30.4)$ & $17(37.0)$ & $15(32.6)$ & \\
\hline$<50000 / \mathrm{mm}^{3}$ & $116(52.02)$ & $22(19.0)$ & $40(34.5)$ & $54(46.6)$ & 0.6 \\
\hline $50000-1.0$ Lakhs $/ \mathrm{mm}^{3}$ & $n^{3} \quad 43(19.28)$ & $10(23.3)$ & $12(27.9)$ & $21(48.8)$ & \\
\hline 1.0Lakh-1.5Lakhs $/ \mathrm{mn}$ & $\mathrm{nm}^{3} \quad 18(8.07)$ & $4(22.2)$ & $7(38.9)$ & $7(38.9)$ & \\
\hline Blast \% & 223 & $50(22.42)$ & $76(34.08)$ & $97(43.50)$ & \\
\hline Mean \pm SD & $63.23 \pm 24.61$ & $62.74 \pm 25.42$ & $63.37 \pm 24.43$ & $63.37 \pm 24.57$ & 0.99 \\
\hline $\mathrm{Hb}$ count $\mathrm{gm} / \mathrm{dl}$ & 223 & $50(22.42)$ & $76(34.08)$ & $97(43.50)$ & \\
\hline Mean \pm SD & $8.44 \pm 2.38$ & $8.49 \pm 2.27$ & $8.70 \pm 2.50$ & $8.31 \pm 2.38$ & 0.57 \\
\hline$>9.0 \mathrm{~g} / \mathrm{dl}$ & $106(47.53)$ & $25(23.6)$ & $39(36.8)$ & $42(39.6)$ & 0.53 \\
\hline$<9.0 \mathrm{~g} / \mathrm{dl}$ & $117(52.47)$ & $25(21.4)$ & $37(31.6)$ & $55(47.0)$ & \\
\hline${ }^{\$} \mathrm{LDH}$ level IU/L & 129 & $30(23.26)$ & $50(38.76)$ & 49(37.98) & \\
\hline Mean \pm SD & $572.19 \pm 493.75$ & $561.63 \pm 608.04$ & $623.20 \pm 506.42$ & $526.61 \pm 400.04$ & 0.62 \\
\hline$<200 I U / L$ & $14(10.85)$ & $5(35.7)$ & $7(50.0)$ & $2(14.3)$ & \\
\hline 200-1000IU/L & $97(75.19)$ & $23(23.7)$ & $32(33.0)$ & $42(43.3)$ & $0.06 \#$ \\
\hline$>1000 \mathrm{IU} / \mathrm{L}$ & $18(13.95)$ & $2(11.1)$ & $11(61.1)$ & $5(27.8)$ & \\
\hline${ }^{\circledR} \mathrm{CR}$ & 168 & $40(23.81)$ & $56(33.33)$ & $72(42.86)$ & \\
\hline $\mathrm{CR}+\mathrm{ve}$ & $100(59.52)$ & $20(20.0)$ & $31(31.0)$ & $49(49.0)$ & $0.09 \#$ \\
\hline CR -ve & $68(40.48)$ & $20(29.4)$ & $25(36.8)$ & $23(33.8)$ & \\
\hline${ }^{\&}$ DFS (months) & 155 & 35 & 50 & 69 & \\
\hline Mean \pm SD & $10.08 \pm 15.08$ & $5.94 \pm 8.37$ & $8.02 \pm 11.056$ & $13.83 \pm 19.09$ & $0.02 *$ \\
\hline
\end{tabular}

only for 168 patients, of which 100 cases were found to be CR +ve (59.52\%) and 68 CR-ve $(40.48 \%)$. The mean disease free survival (DFS) was found to be $10.15 \pm 15.11$ months (0-91months). Based on FAB classification the M2 subtype ( $\mathrm{n}=93(41.70 \%)$ was found to be the most frequent compared to other subtypes.

\section{MDM2-309T>G Genotyping}

The genotype and allele frequencies of MDM2309T $>$ G polymorphism and their odds ratios (Unadjusted and adjusted Odds ratios) under different inherent models with $\chi^{2} \mathrm{p}$ values were represented in Table 2 . MDM2 -309T>G genotype distribution was found to be significantly deviated from Hardy Weinberg equilibrium in AML cases (HWE p<0.001) but not in controls (HWE $\mathrm{p}>0.05)$. The GG genotype and $\mathrm{G}$ allele frequencies were significantly elevated in AML $(\mathrm{p}=<0.0001)$ cases compared to controls under codominant model. Further analysis under various genetic models had revealed that presence of MDM2 -309GG genotype increases the risk for AML by two folds (Adjusted OR=2.18; 95\%CI=1.50-
3.16; $\mathrm{p}=0.0001$ ) under recessive model of inheritance and this association was also found to be similar even after controlling covariates (age and sex) effects. However, -309TG genotype was associated with reduced risk of AML (Adjusted OR=0.52; 95\%CI=0.36-0.74; $\mathrm{p}=0.00004$ ) under overdominant model.

Table 1 shows the genotype distribution with respect to baseline clinical characteristics among AML cases. None showed significant association with MDM2 -309T>G polymorphism in AML, although a borderline significance was observed for GG genotype with elevated LDH level $(>200 \mathrm{IU} / \mathrm{L})(\mathrm{p}=0.06)$ and complete remission rate $(\mathrm{p}=0.09)$. The mean DFS rates were significantly increased in patients with GG genotype $(13.8 \pm 19.1$ months; $p=0.02)$ compared to TT and TG genotypes.

Survival analysis was performed only in patients who have achieved complete remission (CR) after first induction chemotherapy to evaluate the influence of genotypes on the DFS rate of the patients. The median DFS rates in months were represented in Table 3 . The survival analysis revealed that $\mathrm{CR}+\mathrm{ve}$ patients with 
Anuradha Cingeetham et al

Table 2. Genotype Distribution of MDM2 -309T>G Polymorphism among Control and Acute Myeloid Leukemia Cases

\begin{tabular}{|c|c|c|c|c|c|}
\hline \multicolumn{2}{|c|}{ MDM2-309T>G (rs2279744) } & \multicolumn{2}{|c|}{ No: of individuals $(\%)$} & \multicolumn{2}{|c|}{ Adjusted Odds ratios $(95 \% \mathrm{CI})^{@}$} \\
\hline Model of inheritance & Genotype & $\begin{array}{c}\text { Controls (\%) } \\
(\mathrm{N}=304)\end{array}$ & $\begin{array}{l}\text { AML cases }(\%) \\
\quad(\mathrm{N}=223)\end{array}$ & Control vs AML & $\chi^{2} \mathrm{p}$ value \\
\hline \multirow[t]{3}{*}{ Codominant } & TT & $73(24.0)$ & $50(22.4)$ & 1.00 (Ref) & \\
\hline & TG & $150(49.3)$ & $76(34.1)$ & $0.73(0.46-1.16)$ & \\
\hline & GG & $81(26.6)$ & $97(43.5)$ & $1.79(1.12-2.86)^{*}$ & $<0.001 *$ \\
\hline \multirow[t]{2}{*}{ Dominant } & TT & $73(24.0)$ & $50(22.4)$ & $1.00(\operatorname{Ref})$ & \\
\hline & $\mathrm{TG}+\mathrm{GG}$ & $231(76.0)$ & $173(77.6)$ & $1.10(0.73-1.66)$ & \\
\hline \multirow[t]{2}{*}{ Recessive } & $\mathrm{TT}+\mathrm{TG}$ & $223(73.4)$ & $126(56.5)$ & 1.00 (Ref) & 0.66 \\
\hline & GG & $81(26.6)$ & $97(43.5)$ & $2.18(1.50-3.16)^{*}$ & \\
\hline \multirow[t]{5}{*}{ Overdominant } & $\mathrm{TT}+\mathrm{GG}$ & $154(50.7)$ & $147(65.9)$ & 1.00 (Ref) & $0.0001 *$ \\
\hline & TG & $150(49.3)$ & $76(34.1)$ & $0.52(0.36-0.74)^{*}$ & \\
\hline & Allele & & & & $0.0004 *$ \\
\hline & $\mathrm{T}$ & $296(48.7)$ & $176(39.46)$ & 1.00 (Ref) & \\
\hline & G & $312(51.3)$ & $270(60.54)$ & $1.46(1.14-1.86)^{*}$ & $0.003^{*}$ \\
\hline HWE (p) & & $>0.05$ & $<0.001 *$ & & \\
\hline
\end{tabular}

${ }^{\circledR}$ Adjusted (for age and gender) OR and 95\% CI; HWE (Hardy Weinberg equilibrium proportion p-value) $* \mathrm{p}<0.05 ; \# \mathrm{p}<0.10\left(\chi^{2} \mathrm{p}\right.$ values)

Table 3. Genotype Distribution of MDM2 -309T>G Polymorphism and DFS Survival Rate in AML

\begin{tabular}{lccccc}
\hline MDM2-309T>G & $n(\%)$ & $\begin{array}{c}\text { CR+ve (\%) } \\
\text { DFS(Months) } \mathrm{p} \text { value }\end{array}$ & $\begin{array}{c}\text { Median } \\
\text { Dog rank }\end{array}$ \\
\hline Genotype & TT & $34(23.13)$ & $18(52.94)$ & 11 & \\
& TG & $48(32.65)$ & $30(62.50)$ & 9 & $0.05^{*}$ \\
& GG & $65(44.22)$ & $45(69.23)$ & 16 & \\
Total & & 147 & $93(63.27)$ & 11 &
\end{tabular}

Log rank $\mathrm{p}$ values by mantle cox regression $\mathrm{p} \leq 0.05 ; \# \mathrm{p} \leq 0.10$

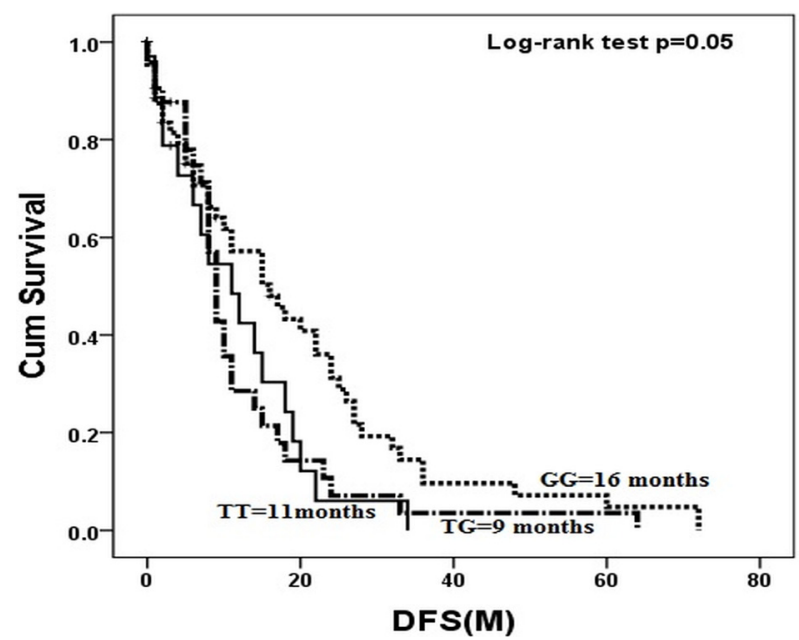

Figure 1. Kaplan-Meier Curves on Disease Free Survival Rates for MDM2-309T>G Polymorphism in AML

MDM2 -309GG genotype had shown increased median survival rate of 16 months as compared to TT (11months) and TG (9 months) genotypes. The log rank test was found to be significant $(\mathrm{p}=0.05)$ (Figure 1$)$.

Real-time analysis of MDM2 mRNA level in AML

To know the effect of MDM2-309T $>$ G polymorphism on the MDM2 expression, we have performed real time PCR analysis in 30 denovo AML patients. MDM2 gene expression was compared with respect to genotypes of MDM2 -309T $>$ G polymorphism. Patients with MDM2 -309GG genotype $(n=15)(0.698 \pm 0.979, p=0.003)$ had shown significantly increased MDM2 mRNA level

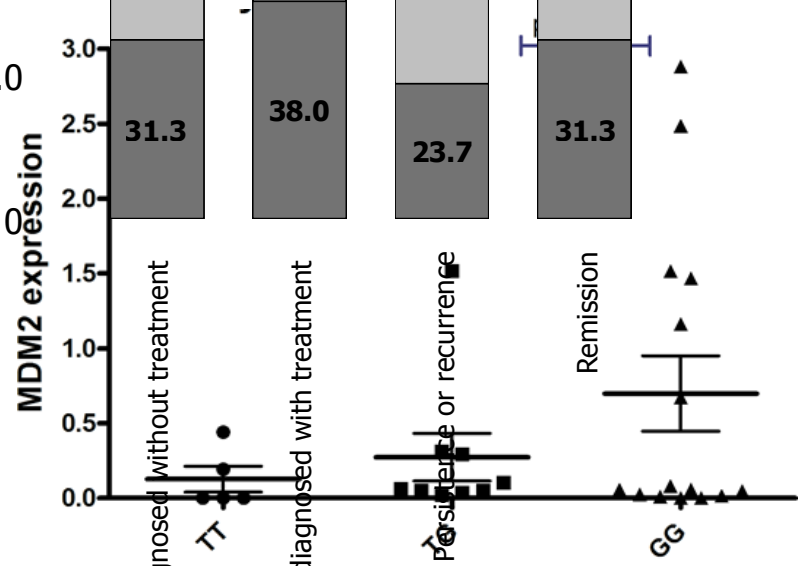

Figure 2. Comparison of MDM2-309T $>$ G Polymorphism and MDM2 mRNA Expression Levels among AML Patients

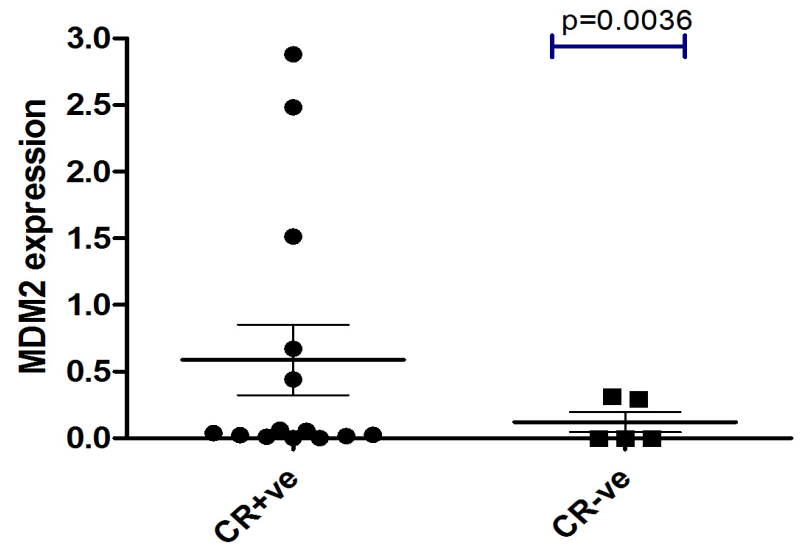

Figure 3. Comparison of MDM2 mRNA Expression Levels with among AML Patients Complete Remission (CR) Rate among AML Patients

compared to TT $(n=5)(0.127 \pm 0.194)$ and TG $(n=9)$ $(0.274 \pm 0.479)$ genotype carriers (Figure 2$)$. Consequently, we have examined the association of MDM2 expression with $\mathrm{CR}$ rate of AML patients after first induction chemotherapy. Interestingly it was observed that MDM2 mRNA levels were significantly elevated in CR+ve cases $(\mathrm{n}=14)(0.587 \pm 0.984 ; \mathrm{p}=0.0036)$ compared to $\mathrm{CR}-\mathrm{ve}$ patients $(n=5)(0.121 \pm 0.166)$ (Figure 3$)$. 


\section{Discussion}

MDM2 is overexpressed in most of the cancers including leukemia (Rayburn et al., 2005) as it is a negative regulator of tumor suppressor $\mathrm{p} 53$, the guardian of the genome. We have studied a promoter polymorphism in MDM2 $(-309 \mathrm{~T}>\mathrm{G})$ to determine the association with AML development and progression as well as to evaluate the functional relationship of this SNP with MDM2 expression levels in AML. The present study had shown a significant association of MDM2 -309T $>$ G polymorphism with AML development. The -309G allele carriers were found to have 1.46 folds of risk of AML compared to $\mathrm{T}$ allele carriers. Further, our study did not show significant association of MDM2-309T $>$ G polymorphism with epidemiological variables (age at onset, gender) and clinical parameters like WBC count, platelet count, hemoglobin level, peripheral blast $\%$ in AML. These findings were in accordance with previous studies by Xiong et al (2009) and Phillips et al (2010) who had also reported a significant association of GG genotype with AML development in adults $(\mathrm{OR}=3.52$; $\mathrm{p}=0.000)$ and children $(\mathrm{OR}=1.5 ; \mathrm{p}=0.049)$ respectively, but not with any clinical parameters. A meta-analysis study conducted on 66 case control studies with respect to MDM2 -309T>G polymorphism had provided evidence that the presence of homozygote $-309 \mathrm{GG}$ and heterozygote -309TG were significantly associated with increased risk for all tumors which was more evident in Asian and European populations (Wan et al., 2011). Moreover, our results are in concordance with a meta-analysis conducted exclusively in leukemia which demonstrated association of MDM2 -309T>G polymorphism especially with Asian population (Yan et al., 2014). Further, our study had revealed that CR+ve cases with MDM2 -309GG genotype had significantly elevated DFS rates compared to $\mathrm{CR}+\mathrm{ve}$ TT and TG genotype groups.

Real time PCR analysis indicated a significantly increased MDM2 expression in GG genotype carriers compared to TT and TG genotype. Our results are in accordance with Hong et al (2005) and Xiao et al (2010), where MDM2 -309GG genotype carriers were found to be associated with significantly elevated MDM2 mRNA level in Esophageal Squamous Cell Carcinoma (ESCC) and nasopharyngeal carcinoma (NPC) compared to other genotypes. Our results suggest that GG genotype with increased expression of MDM2 might lead to inhibition of TP53 function increasing the risk to leukemiogenesis. Further, MDM2 levels were also elevated in CR+ve cases. Thus, it can be concluded that presence of GG genotype at promoter region (MDM2 $-309 \mathrm{~T}>\mathrm{G}$ ) might increase MDM2 gene expression, thereby inhibiting the p53 stress response resulting in leukemic cell transformation. The association of GG genotype and elevated MDM2 expression with increased DFS and complete remission rates suggest that the overexpression of MDM2 may lead to cell vulnerability to chemotherapy in absence of regulation of cellular machinery due to Tp53 degradation. Similarly, a study on primary gastric cancer patients from Chinese population have reported that high MDM2 expression in tumor tissue had significantly elevated survival rates who are on adjuvant FLO chemotherapy (Ye et al., 2013).

In summary, our results suggest that MDM2 overexpression among GG carriers might increase risk to AML development. Increased MDM2 mRNA expression level at diagnosis might elevate $\mathrm{CR}$ rate after first induction therapy among AML patients.

\section{Acknowledgements}

University Grants Commission, New Delhi, India had supported this study by providing financial assistance to SV through Emeritus fellowship and Indian Council for Medical Research (ICMR), New Delhi, had encouraged AC to carry out this work by awarding SRF. We sincerely acknowledge all the subjects and healthy volunteers for participation in the study. We are very much thankful to all the people who had contributed their effort in completing this work.

Conflict of Interest Statement: Authors declared no conflicts of interests.

\section{References}

Barak Y, Gottlieb E, Juven-Gershon T, Oren M (1994). Regulation of mdm2 expression by p53: Alternative promoters produce transcripts with nonidentical translation potential. Genes Dev, 8, 1739-49.

Bond GL, Hu W, Bond EE, Robins H, Lutzker SG, Arva NC, et al (2004). A single nucleotide polymorphism in the MDM2 promoter attenuates the $\mathrm{p} 53$ tumor suppressor pathway and accelerates tumor formation in humans. Cell, 119, 591-602.

Boyd MT, Vlatkovic N, Haines DS (2000). A novel cellular protein (MTBP) binds to MDM2 and Induces a G1 arrest that is suppressed by MDM2. J Bio Chern, 275, 31883-90.

Brown CY, Mize GJ, Pineda M, George DL, Morris DR (1999). Role of two upstream open reading frames in the translational control of oncogene mdm2. Oncogene, 18, 5631-7.

Cheng TH, Cohen SN (2007). Human MDM2 isoforms translated differentially on constitutive versus p53-regulated transcripts have distinct functions in the p53/MDM2 and TSG101/ MDM2 feedback control loops. Mol Cell Biol, 27, 111-9.

Ebid GT, Sedhom IA, El-Gammal MM, Moneer MM (2012). MDM2 T309G has a synergistic effect with P21 ser31arg single nucleotide polymorphisms on the risk of acute myeloid leukemia. Asian Pac J Cancer Prev, 13, 4315-20.

Ellis NA, Huo D, Yildiz O, et al (2008). MDM2 SNP309 and TP53 Arg72Pro interact to alter therapy-related acute myeloid leukemia susceptibility. Blood, 112, 741-9.

Hong Y, Miao X, Zhang X, et al (2005). The Role of P53 and MDM2 polymorphisms in the risk of esophageal squamous cell carcinoma. Cancer Res, 65, 9582-7.

Lahiri DK and Nurnberger JI (1991). A rapid non-enzymatic method for the preparation of HMW DNA from blood for RFLP studies. Nucleic Acids Res, 19, 5444.

Michael D, Oren M (2002). The p53 and Mdm2 families in cancer. Curr Opin Genet Dev, 12, 53-9.

Montes de Oca Luna R, Tabor AD, Eberspaecher H, Hulboy DL, Worth LL, et al (1996). The organization and expression of the mdm2 gene. Genomics, 33, 352-7.

Oliner JD, Kinzler KW, Meltzer PS, George DL, Vogelstein B (1992). Amplification of a gene encoding a p53-associated protein in human sarcomas. Nature, 358, 80-3.

Phillips CL, Gerbing R, Alonzo T et al (2010). MDM2 polymorphism increases susceptibility to childhood acute 
Anuradha Cingeetham et al

myeloid leukemia: a report from the children's Oncology Group. Pediatr Blood Cancer, 55, 248-53.

Rayburn E, Zhang R, He J, Wang H (2005). MDM2 and human malignancies: Expression, clinical pathology, prognostic markers, and implications for chemotherapy. Curr Cancer Drug Targets, 5, 27-41.

Wan Y, Wu W, Yin Z, Guan P, Zhou B (2011). MDM2 SNP309, gene-gene interaction, and tumor susceptibility: an updated meta-analysis. BMC Cancer. 11, 208.

Xiao M, Zhang L, Zhu X, et al (2010). Genetic polymorphisms of MDM2 and TP53 genes are associated with risk of nasopharyngeal carcinoma in a Chinese population. BMC Cancer, 10, 147.

Xiong X, Wang M Wang L, et al (2009). Risk of MDM2SNP309 alone or in combination with the $\mathrm{p} 53$ codon 72 polymorphism in acute myeloid leukemia. Leuk Res, 33, 1454-8.

Wan Y, Wu W, Yin Z, Guan P, Zhou B (2011). MDM2 SNP309, gene-gene interaction, and tumor susceptibility: an updated meta-analysis. BMC Cancer. 11, 208.

Yan YL, Han F, Tan WM, Wu CP, Qin X (2014). Association between the MDM2 T309G polymorphism and leukemia risk: a meta-analysis. Asian Pac J Cancer Prev, 15, 6767-72.

Ye Y, Li X, Yang J, et al (2013). MDM2 is a useful prognostic biomarker for resectable gastric cancer. Cancer Sci, 104, 590-98.

Zauberman A, Flusberg D, Haupt Y, Barak Y, Oren M (1995). A functional p53-responsive intronic promoter is contained within the human mdm2 gene. Nucleic Acids Res, 23, 2584-92. 